\title{
Effect of surfactants on the fluorescence spectra of water-soluble MEHPPV derivatives having grafted polyelectrolyte chains
}

\author{
NAGESH KOLISHETTI and S RAMAKRISHNAN* \\ Department of Inorganic and Physical Chemistry, Indian Institute of Science, Bangalore 560012 \\ e-mail: raman@ipc.iisc.ernet.in
}

\begin{abstract}
Poly(2-methoxy-5-[2'-ethylhexyoxy]-1,4-phenylenevinylene) (MEHPPV) derivatives with polyacrylic acid (PAA) chains grafted onto their backbone were found to be water soluble, and they exhibited a dramatic increase in their fluorescence intensity in the presence of a variety of surfactants, even at concentrations far below their critical micelle concentrations (CMC). This increase was accompanied by a blue-shift in the emission maximum. These observations are rationalized based on the postulate that the backbone conformation of the conjugated polymer is modulated upon interaction of the surfactant molecules with the polyelectrolytic tethers, which in turn results in a significant depletion of intra-chain interchromophore interactions that are known to cause red-shifted emission bands with significantly lower emission yields.
\end{abstract}

Keywords. Fluorescence spectra; MEHPPV derivatives; grafted polyelectrolyte chains.

\section{Introduction}

Conjugated polymers (CP) have been the focus of investigations because of their potential for application in a variety of devices, such as light emitting diodes (LED), field effect transistors (FET), photovoltaics etc. ${ }^{1-4}$ Extensive research to modulate the photophysical properties of CP's in solution in the presence of small molecules has been carried out by various groups over the last decade.$^{5-11}$ Primary focus has been on water-soluble derivatives of conjugated polymers that possess ionic groups in their backbone, namely conjugated polymer electrolytes (CPE), especially because of their biological relevance. For metal sensing, simple organic soluble CP's were used, ${ }^{5,6}$ while for biological sensors, water-soluble CPE's have been shown to hold great promise. ${ }^{7-9}$ Several examples describe the incorporation of new functional handles onto the conjugated polymer backbone so that they can interact with small-molecule analytes and in turn cause modulation of their fluorescence properties. ${ }^{12-19}$ Whitten and coworkers have demonstrated that conjugated polymer-based polyelectrolytes can be designed to exhibit unprecedented sensitivity to specific analytes, to such an extent that even a single binding event on a chain can lead to complete quenching of fluorescence from the entire backbone. $^{7}$

*For correspondence
Most of the water-soluble conjugated polymers studied thus far derive their solubility from the presence of a large number of regularly placed ionic groups along their backbone. We have recently explored an alternate design wherein polyelectrolyte chains have been grafted onto the backbone of a conjugated polymer, namely poly (2-methoxy-5-[2'ethylhexyoxy]-1,4-phenylenevinylene) (MEHPPV). ${ }^{20}$ To achieve grafting, we utilized an appropriate precursor (MDP- $x$ ) that contains potential free-radical initiating sites, namely benzyl dithiocarbamate (DTC), which are randomly distributed along the polymer backbone (see scheme 1). These and other similar precursor copolymers were initially prepared by us as an approach to control the average molecular conjugation length in these so-called segmented MEHPPV's, which in turn served to modulate the colour of emission from these polymers. ${ }^{21-23}$ An additional feature of MDP-x precursors, is the presence of benzyl dithiocarbamate units, which are known to function as iniferters ${ }^{24,25}$ - a term that implies that they can simultaneously serve as initiators, a chain-transfer agents and terminators of free-radical polymerizations. UV irradiation is the trigger to activate the polymerization process using iniferters. The benzyl radical that is generated upon irradiation serves as an initiator for the polymerization, while the relatively stable sulphurbased radical reversibly terminates the growing polymer chain by recombination as depicted in scheme 2. Based on this mechanism, the DTC groups in MDP- $x$ 


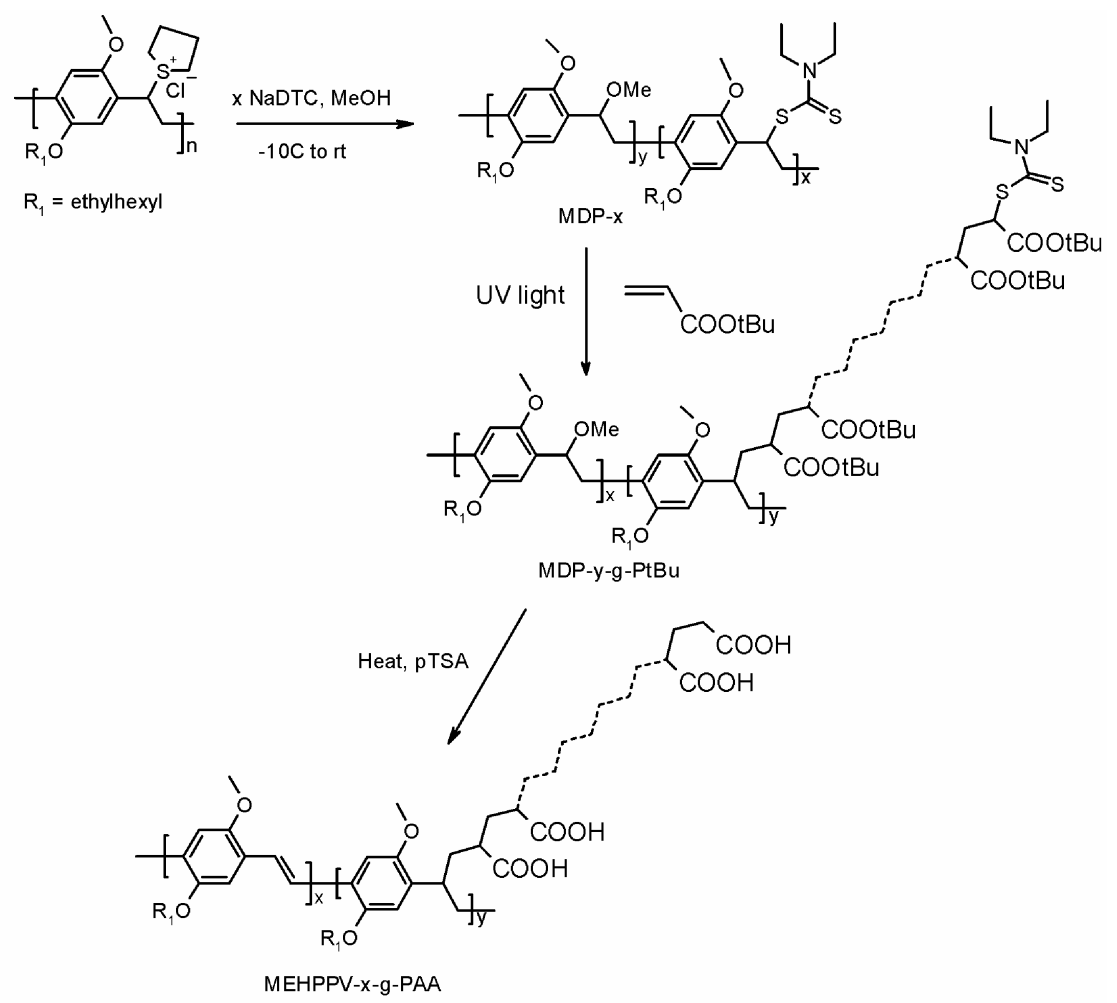

Scheme 1. Synthetic methodology for grafting various monomers onto the backbone of MDP- $x$.<smiles>[R]C(CCCc1ccccc1)[R](C)(C)CCc1ccccc1</smiles>

Scheme 2. Polymerization of monomer with BDTC (A) as iniferter.

precursors were utilized to initiate the polymerization of a variety of monomers, including $t$-butylacrylate. ${ }^{20}$ The resulting graft copolymers containing residual methoxy groups were subject to acid-catalysed thermal elimination to generate the conjugated MEHPPV derivative as depicted in scheme 1. Interestingly, during this catalysed thermal elimination process the de-protection of the $t$-butyl ester groups was also achieved, yielding a MEHPPV derivative possessing ionizable polyacrylic acid (PAA) chains grafted to its backbone, and thereby rendering them soluble in aqueous base. ${ }^{20}$

In this paper, we describe the fluorescence spectroscopic studies of these novel water-soluble MEHPPV derivatives (MEHPPV-60-g-PAA) containing grafted polyacrylic acid chains. Specifically, we examine the effect of various surfactants on their fluorescence spectra, which in turn sheds light on the conformational changes that are induced by the interaction of the surfactants with the polyelectrolytic tethers.

\section{Experimental section}

\subsection{Materials}

Synthesis of the MDP- $x$ precursor copolymers containing controlled amounts of randomly distributed dithiocarbamate units was described in our previous report. ${ }^{20}$ $t$-Butyl acrylate, and $p$-toluenesulphonic acid ( $p$ TSA), dodecyl trimethyl ammonium bromide $\left(\mathrm{C}_{12} \mathrm{TAB}\right)$, myristyl trimethyl ammonium bromide $\left(\mathrm{C}_{14} \mathrm{TAB}\right)$, cetyl trimethyl ammonium bromide $\left(\mathrm{C}_{16} \mathrm{TAB}\right)$, octadecyl trimethyl ammonium bromide $\left(\mathrm{C}_{18} \mathrm{TAB}\right)$ were purchased from Aldrich. Sodium chloride, calcium chloride and Triton-X-100 were purchased from Sd Fine Chemicals. Acrylate monomers were purified according to the standard procedures. ${ }^{26}$ Tetrahydrofuran, chloroform, dichloromethane and methanol were dried by refluxing over sodium, phosphorous pentoxide and magnesium turnings, respectively. All the solvents were distilled freshly before use. 


\subsection{Instrumental methods}

NMR spectra of the polymers were recorded on a Bruker $400 \mathrm{MHz}$ spectrometer. UV-visible and fluorescence spectra were recorded on Perkin-Elmer Lambda 35 UV-visible spectrometer and PerkinElmer LS-50B fluorescence spectrophotometer, respectively. An excitation wavelength of $370 \mathrm{~nm}$ was used for the latter. Time-resolved fluorescence studies were carried out on an IBH work station, having a full-width at half maximum of the lamp of $\approx 1.9$ $1.4 \mathrm{~ns}$. The response time of the detector was about 150 ps. Gel Permeation Chromatography (GPC) measurements were carried out with a Viscotek TDA model 300-detector system and a set of PL gel mixed-bed columns for separation uning THF as the eluent at $30^{\circ} \mathrm{C}$. Molecular weights were determined with a conventional calibration curve constructed with polystyrene standards in THF at $30^{\circ} \mathrm{C}$. An UVI-Tec $12 \mathrm{~W}$ UV lamp with monochromatic light wavelength of $365 \mathrm{~nm}$ was used for photo-initiation of the graft polymerization.

\subsection{Fluorescence measurements}

All the FL measurements were carried out using aqueous or methanolic solutions. For all studies in aqueous media, polymer solutions were prepared by dissolving MEHPPV-60-g-PAA in water containing one equivalent of $\mathrm{NaOH}$ with respect to PAA repeat unit; this is to ensure complete neutralization of the carboxylic acid groups and render the polymer soluble. For a typical fluorescence measurement a stock solution of MEHPPV-60-g-PAA ( $10 \mathrm{mM}$ w.r.t. to PAA) was used. Stock solutions of the surfactants with concentrations ranging from 2 to $10 \mathrm{mM}$ were prepared depending on the requirement. To the solution of the polymer required amounts of the surfactant stock solution was added using a micropipette. The resultant spectra were then normalized to take into account the dilution upon addition of the surfactant solution. All the measurements were carried out using $430 \mathrm{~nm}$ excitation. For quantitative comparison of the emission intensity, the area under the fluorescence envelop was used.

\section{Results and discussion}

The synthesis of the MDP- $x$ precursors and the grafting of t-butyl acrylate onto their backbone were carried out as described earlier. ${ }^{20}$ Briefly, the MDP- $x$ precursors containing the required amount of DTC groups (in this case, MDP-40 containing $40 \mathrm{~mol} \%$ of DTC units and the remaining methoxy units) was dissolved in THF along with the required amount of $t$-butyl acrylate monomer in a polymerization tube and sealed under vacuum after degassing. The sealed tubes were exposed to UV light for $90 \mathrm{~min}$ after which the polymer was isolated. GPC analysis of the resulting polymers shows a substantial increase in the molecular weight $\left(M_{n}\right)$ from 104700 to 998000 . Significant amounts of the homopolymer, polybutyl acrylate, was also formed; a portion of which was readily removed by fractionation. Complete removal of the homopolymer, however, was found to be difficult, but this was not of serious concern for the present study as PAA chains are effectively silent to the fluorescence measurements, especially at the very low concentrations that were typically used. The acid-catalysed thermal elimination of the precursor yielded the conjugated polymer, MEHPPV-60-gPAA, wherein complete hydrolysis of $t$-butyl ester groups of the grafted poly( $t$-butyl acrylate) had occurred; here 60 refers to the expected mole-percent of conjugated units formed upon complete elimination of the methoxy groups. The disappearance of the $t$-butyl groups in the proton NMR spectrum confirmed the complete hydrolysis. The eliminated polymers were completely soluble in aqueous alkaline medium and also in polar solvents, such as methanol/ethanol. This implied that a significant extent of grafting of water-soluble PAA chains onto the MEHPPV backbone had indeed occurred. If, however, the grafting reaction time was reduced, the final eliminated/hydrolyzed product was found to be insoluble in aqueous base/alcohol, confirming the importance of adequate levels of grafting for imparting solubility in polar solvents.

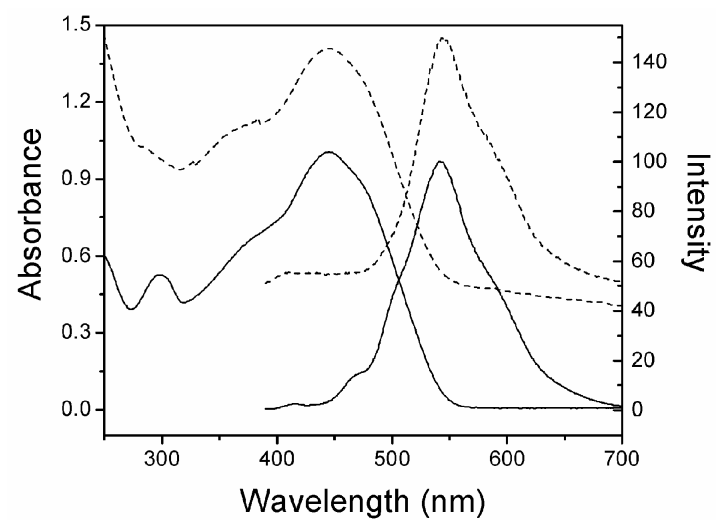

Figure 1. UV-visible and FL spectra of MEHPPV-60g-PAA (dashed line) in methanol and MEHPPV-58 (solid line) in dichloromethane. ${ }^{27}$ 
The absorption and fluorescence (FL) spectra of the eliminated polymers, MEHPPV-60-g-PAA, in methanol are shown in figure 1 . As evident from the figure, these spectra resemble those of MEHPPV-58, wherein $58 \mathrm{~mol} \%$ of the repeat units are conjugated while the remaining are non-conjuated. ${ }^{27}$ This is accordance with our expectation based on the fact that once the DTC groups are utilized to initiate the grafting, only the remaining methoxy groups (60 $\mathrm{mol} \%$ in the case of MDP-40 precursor) are removed during the acid-catalysed thermal elimination process. This would generate a backbone consisting of $60 \mathrm{~mol} \%$ of eliminated units while the remaining sites would have grafted PAA chains. Time-resolved fluorescence studies of the grafted MEHPPV-60-gPAA yielded a life time around $0.5 \mathrm{~ns}$, which is similar to the life-time value of the nascent MEHPPV-x. ${ }^{28}$ While the proton NMR spectra clearly indicate the presence of grafted poly(t-butyl acrylate) chains in the precursor, the composition of these clearly suggest the presence of un-grafted homopolymer even after fractionation, and hence precise quantification of the grafting levels was difficult. However, clear evidence for grafting having occurred from the DTC sites was evident in the NMR spectrum of the grafted precursor, wherein a near complete disappearance of the benzylic protons adjacent to the DTC group was observed. ${ }^{27}$

Having established the structure of the watersoluble MEHPPV-g-PAA polymer, we decided to examine the effect of various analytes, that could interact with the grafted polyacrylic acid (PAA) chains, on the fluorescence spectrum arising from the conjugated backbone. We showed earlier that quenchers, such as methyl viologen, cause a dramatic reduction in the emission yield as do some metal ions, like $\mathrm{Cu}^{2+} \cdot{ }^{20}$ Previous studies by other researchers have indicated that CPE's could exhibit very distinctly different behaviour in the presence of oppositely charged ionic surfactants - both enhancement in the fluorescence intensity ${ }^{10,18,29}$ as well as quenching of fluorescence have been reported. ${ }^{30}$ The enhancement in fluorescence was ascribed to the disruption of aggregates due to surfactant condensation onto the polyelectrolyte backbone in the case of relatively flexible PPV derivatives, ${ }^{10}$ while in the case of stiff polyfluorene-based oligomeric polyelectrolytes, surfactant-induced aggregation was shown to be responsible for quenching. ${ }^{30}$ Interestingly, in the case of polyfluorene-based systems, the same authors also reported an increase in the presence of nonionic surfactants, at concentrations above their $\mathrm{CMC}^{29}$ This enhancement was ascribed to micellar entrap- ment of conjugated polymer chains leading to aggregate disruption and consequent enhanced fluorescence. All the above studies were carried out using CPE's wherein the ionic groups are present at periodic intervals along the conjugated polymer backbone. The diametrically opposite responses observed in CPE systems in the presence of different types of surfactants clearly points to the complex origins for the observed spectral changes. Our grafted-MEHPPV derivatives differ from those CPE's examined earlier in two ways - (a) the approach used for preparing these polyelectrolyte grafted systems creates segmented conjugation along the backbone and thereby introduces a statistical distribution chromophores with different conjugation lengths (excitation and emission energies), and (b) the polyelectrolyte chains carrying a large number of ionic functionality is tethered at random locations along the conjugated polymer backbone. Both these molecular architectural differences suggest that the response we might observe in the presence of surfactants could be distinctly different from those earlier observed. With this in mind, we examined the effect of different cationic surfactants having varying alkyl chain lengths, in addition to one anionic and one non-ionic surfactant.

\subsection{Effect of cationic surfactants}

Figure 2 shows the effect of cetyl trimethylammonium bromide $\left(\mathrm{C}_{16} \mathrm{TAB}\right)$ on the FL spectra of MEHPPV60-g-PAA. The FL intensity increases with increase in the concentration of $\mathrm{C}_{16} \mathrm{TAB}$ and then levels-off

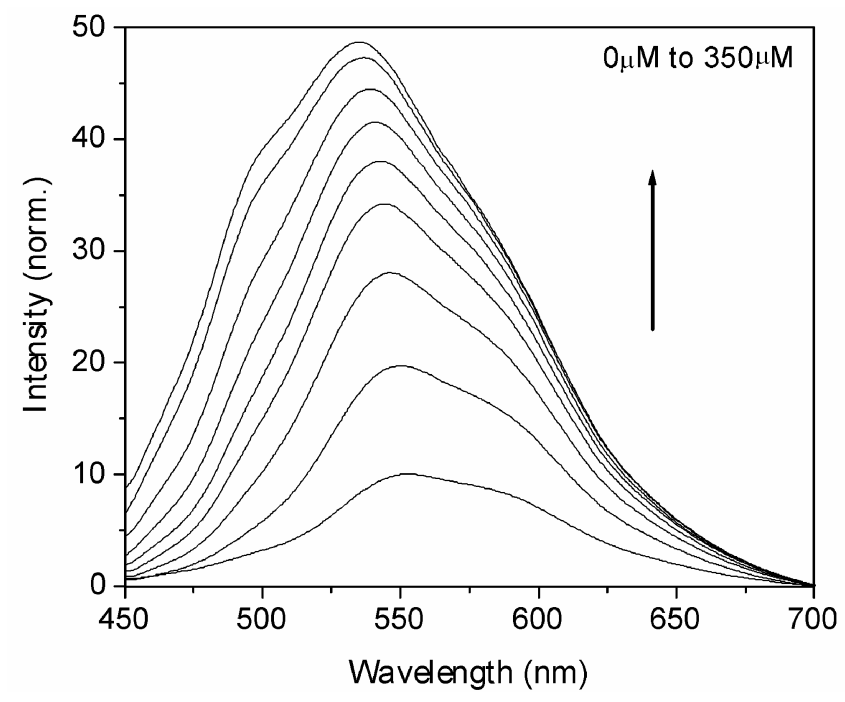

Figure 2. Effect of $\mathrm{C}_{16} \mathrm{TAB}$ concentration on $\mathrm{FL}$ of MEHPPV-60-g-PAA in water. All the spectra were concentration normalized ( $1 \mathrm{mM}$ w.r.t PAA). 
beyond a certain concentration. Along with the dramatic increase in the fluorescence intensity, two other features are also evident: one is a gradual blue-shift of the emission maximum and the other is the growth of a shoulder at lower wavelength, around $500 \mathrm{~nm}$. This suggests that the interaction of the surfactant with the PAA chains appears to cause an expansion of the coil and thereby reduces the intra-chain interchromophore interactions. Such effects were earlier reported by us in segmented MEHPPV's as a function of solvent composition; going from a poor solvent to a good solvent caused a similar change in the fluorescence spectra. ${ }^{28}$ Further discussions of these observations will follow later.

As the experiment was performed by step-wise addition of a surfactant solution to an aqueous solution of the conjugated polymer, the effect of dilution of the conjugated polymer must be factored-in; thus plots shown are normalized to a constant concentration ( $1 \mathrm{mM}$ w.r.t. the PAA repeat unit) of the polymer. It may be added here that since the exact composition of the MEHPPV-60-g-PAA is not readily determinable due to the presence of PAA homopolymer, the concentrations were taken with respect to PAA repeat unit (majority component in the sample), assuming that the fraction of MEHPPV-g-PAA in the sample is homogeneous and invariant. Furthermore, the absorbance (OD) at the maximum of the MEHPPV segment during all the measurements was maintained roughly constant $(<0 \cdot 1)$ to ensure that the concentrations of the MEHPPV-60-g-PAA was in the same region for all the measurements. Similar normalization of the absorption spectra to reflect constant concentration (figure 3), demonstrated very little

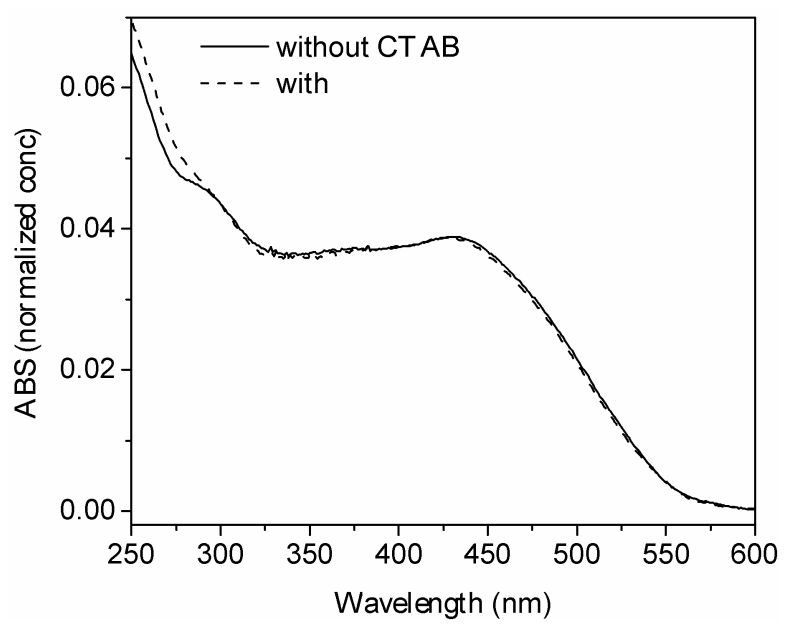

Figure 3. Concentration ( $1 \mathrm{mM}$ w.r.t. PAA) normalized absorption spectra of MEHPPV-60-g-PAA without and with $350 \mu \mathrm{M} \mathrm{C}_{16} \mathrm{TAB}$. variation its shape and intensity, suggesting that the effect of surfactant is primarily on the chain conformation rather than due to a break-up of ground state aggregates, contrary to earlier observations by Chen et al ${ }^{10}$.

The variation of the normalized area under the emission envelop is plotted as a function of the $\mathrm{C}_{16} \mathrm{TAB}$ concentration in figure 4 , along with variation in the $\lambda_{\max }$ value. Firstly, it is evident that both these variations are a reflection the same underlying process, as the variation is similarly steep in the beginning and levels-off beyond a certain concentration; in other words the variation of emission intensity mirrors that seen in the $\lambda_{\max }$ values. It must be noted that the saturation occurs at much lower concentrations $(\sim 50 \mu \mathrm{M})$ when compared to the CMC of $\mathrm{C}_{16} \mathrm{TAB}$, which was determined to be ca. $800 \mu \mathrm{M} \cdot{ }^{31}$ Condensation of oppositely charged surfactants onto polyelectrolyte chains is a well-studied phenomenon and is known to dramatically modify chain conformations. ${ }^{32-35}$ A similar effect is likely to be responsible for the dramatic increase in the fluorescence intensity induced by backbone conformational modulation.

It is important to recall here that the precursor onto which grafting has been effected contains $40 \mathrm{~mol} \%$ of DTC groups, all of which appear to have been utilized to initiate grafting; as was evident from our earlier comparison of the fluorescence spectrum of MEHPPV-60-g-PAA with that of MEHPPV58 (figure 1). Such segmented MEHPPV's contain a large number of chromophores having a distribution

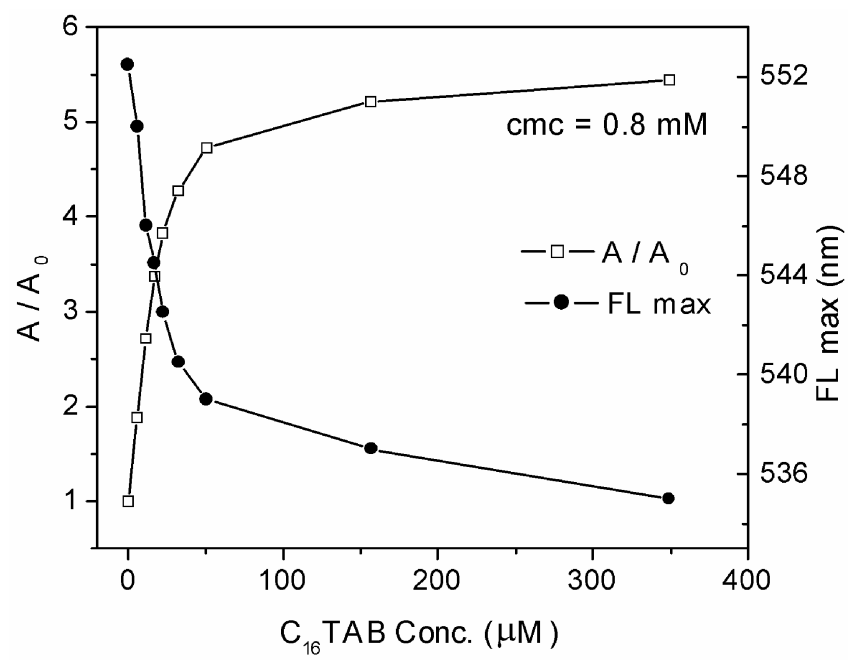

Figure 4. Variation of normalized area under emission spectra (A/Ao) and FL $\lambda_{\max }$ of MEHPPV-60-g-PAA as a function of $\mathrm{C}_{16} \mathrm{TAB}$ concentration. 
of conjugation lengths and have been shown to exhibit substantial degree of energy transfer, the extent of which is depends very strongly on the backbone conformation. ${ }^{28}$

In an effort to gain further insight into this process, we compare the surfactant-induced variation seen in MEHPPV-60-g-PAA to the solvent-induced varia tion of the fluorescence spectra of a segmented

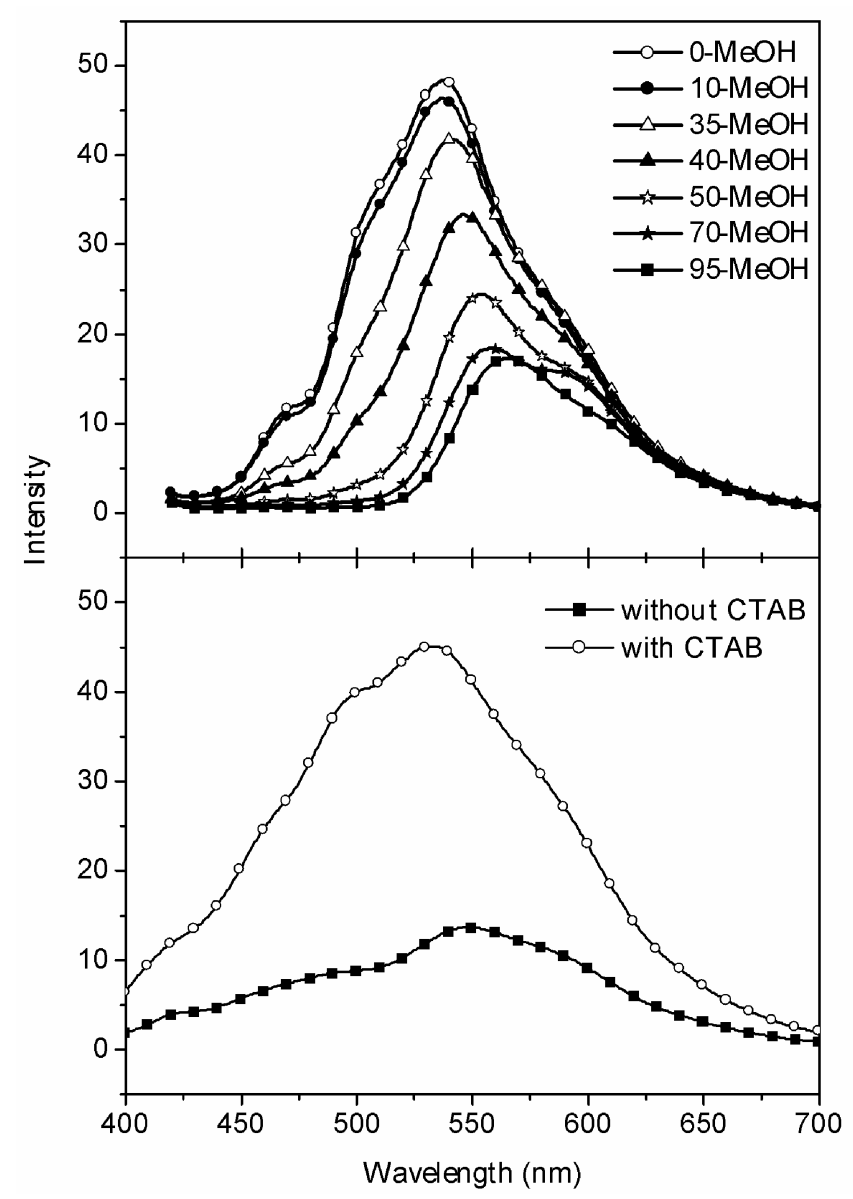

Figure 5. Concentration normalized emission spectra of MEHPPV-60-g-PAA in the absence and presence of $350 \mu \mathrm{M}$ of $\mathrm{C}_{16} \mathrm{TAB}$ (bottom). Variation of FL spectra of MEHPPV-55 as a function of solvent composition (methanol : DCM) (top) ${ }^{28}$.

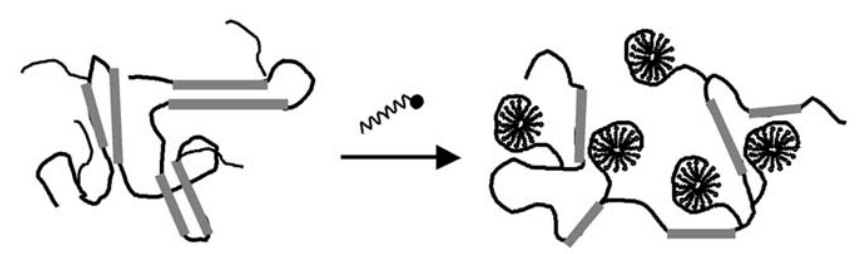

Scheme 3. A schematic depiction of the process of separation of the segmented chromophores along the backbone in the presence of a surfactant.
MEHPPV-x with the nearest composition; namely MEHPPV $-55^{28}$ (figure 5). Although the solvents used in these studies are different - water in the present study along with surfactant, but dichloromethane (DCM)-methanol mixtures in our earlier investigation, the similarity, in terms of intensity, emission maxima and line shape, is rather striking. This comparison of the spectral profiles appears to suggest that the conformation of MEHPPV-60-gPAA in water is similar to the compact conformation adopted by MEHPPV-55 in roughly $70: 30(v / v)$ methanol:DCM, although some differences in the blue-region of the spectrum are noticeable. On the other hand, in the presence of $350 \mu \mathrm{M}$ concentration of $\mathrm{C}_{16} \mathrm{TAB}$, the conformation of MEHPPV-60-gPAA is similar to that of MEHPPV-55 in a good solvent, like pure DCM. This is a remarkable observation that suggests that even at such a low concentration of the surfactant one is able to affect a dramatic change in the conformation of the segmented MEHPPV-60 backbone, because of specific interactions of the surfactants with the grafted polyelectrolyte chains. A schematic depiction of the process of unfolding of the collapsed chain in the presence of a surfactant is shown in scheme 3. It is important to recall that this coil expansion leads to less effective energy transfer within a single chain in addition to a depletion in the number of intra-chain inter-chropmophore excitions, both of which lead to a blue-shifted emission with enhanced intensity. The shoulder in the blue is further evidence for direct emission from shorter conjugation length chromophoric units, prior to energy transfer to higher conjugation length chromophores. This reduction in efficacy of energy transfer is due to the higher average inter-chromophore distance in the expanded chain conformation. ${ }^{28}$

Similar FL studies were carried out in the presence of various other cationic surfactants, with varying alkyl chain lengths, from $\mathrm{C} 12$ to $\mathrm{C} 18$. In all cases, a similar enhancement in the emission intensity with a slight blue-shift in the emission maxima is observed. In figure 6 , we compare the variations in the normalized intensities and FL $\lambda_{\max }$ values in the presence of various surfactants. It is clear that in all cases one sees an initial rapid increase followed by saturation at higher concentrations; importantly, the saturation occurs at concentrations significantly lower than their respective CMC's (listed in the respective plots). Here again, it is observed that the variations in intensity parallels that of the $\lambda_{\max }$ values confirm- 
ing that these variations reflect the same underlying process. Attempts to correlate the hlb (or CMC) of the surfactant with some specific feature of the
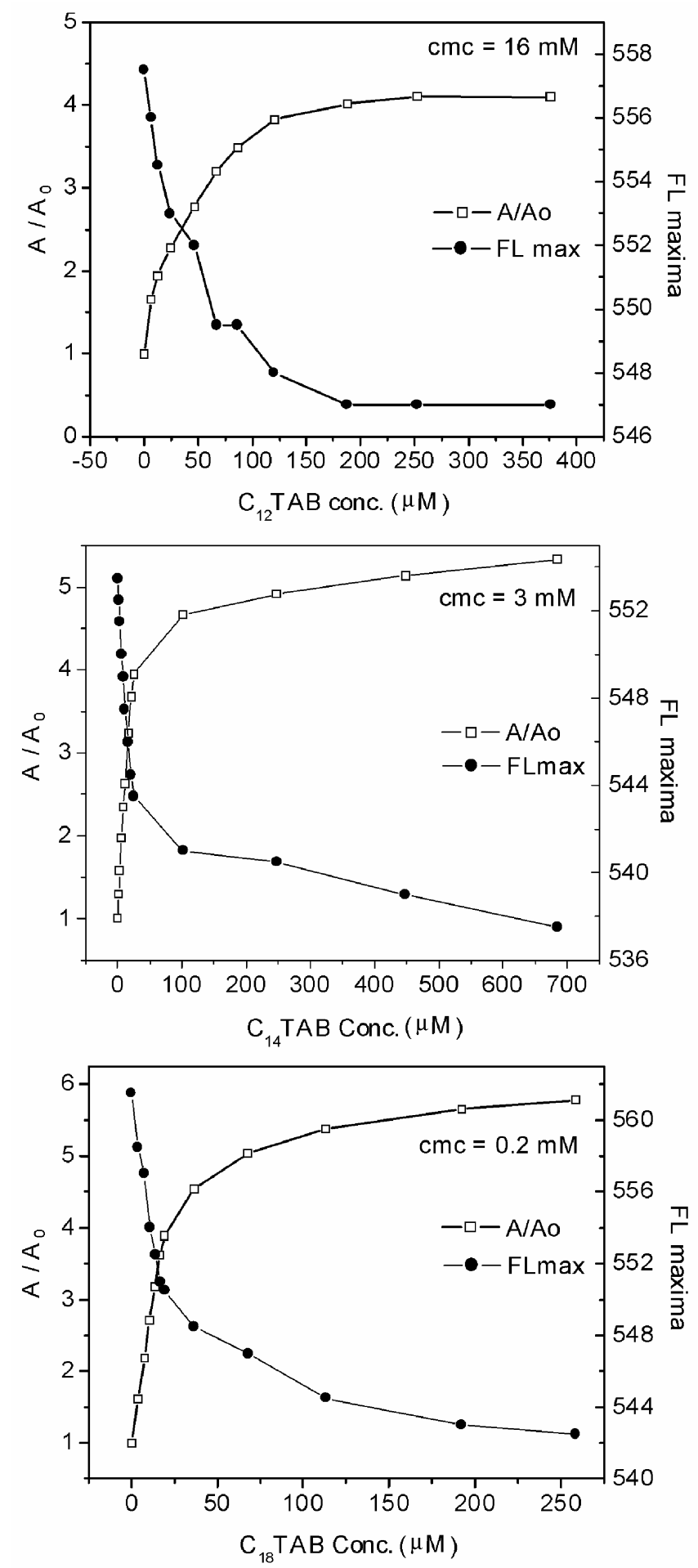

Figure 6. Comparison of variation of area under emission spectra (A/Ao) of MEHPPV(60)-g-PAA for various surfactants. The CMC values listed in each plot were determined by conductometric titration. variation profile, such as the saturation enhancement or the initial slope, did not yield any meaningful insight.

In order to confirm the importance of surfactancy, the fluorescence spectra of MEHPPV-60-g-PAA were similarly monitored in the presence of a simple organic ammonium salt, namely tetraethyl ammonium chloride (TEAC). Figure 7 shows the variation in FL spectra as a function of increasing TEAC concentration: Note that the maximum concentration used here $(7.2 \mathrm{mM})$ is an order of magnitude higher than in the case of the surfactants. The enhancement noticed even at such high concentrations is only around 1.4 fold, which clearly illustrates the impor-

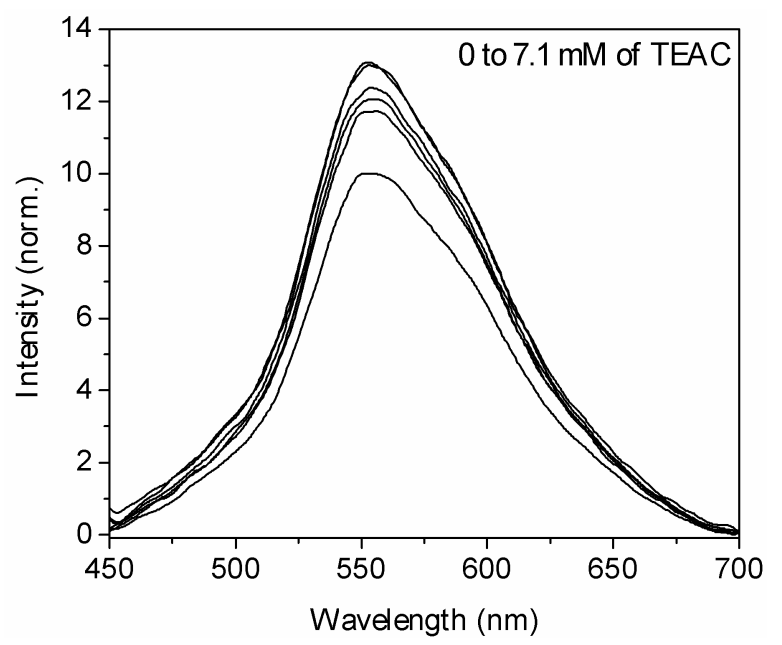

Figure 7. Effect on FL of MEHPPV(60)-g-PAA with variation of tetraethyl ammonium chloride (TEAC) in the medium. The spectra are concentration normalized (1 mM w.r.t. PAA).

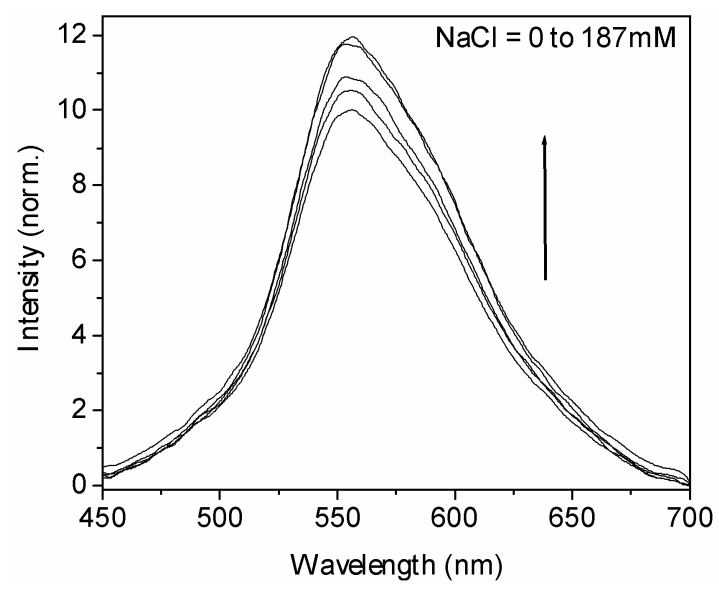

Figure 8. Effect of sodium chloride $(\mathrm{NaCl})$ on $\mathrm{FL}$ of MEHPPV-60-g-PAA. The spectra are concentration normalized ( $1 \mathrm{mM}$ w.r.t. PAA). 
tance of surfactancy for the observed enhancement in FL emission. Similarly, when the titration was carried out in methanol, very little change in the FL spectra was observed (not shown) reconfirming the importance of surfactancy.

The small increase in the emission intensity in the presence of TEAC could also be because of an increase in the concentration of sodium chloride, which gets liberated when the cationic ammonium group displaces the sodium from the sodium acrylate chains. To examine the effect of ionic strength, we directly added sodium chloride solution to the solution of MEHPPV-60-g-PAA, which should result in a similar change in ionic strength of the medium. From figure 8 , it is evident that sodium chloride has a similar effect as that of TEAC.

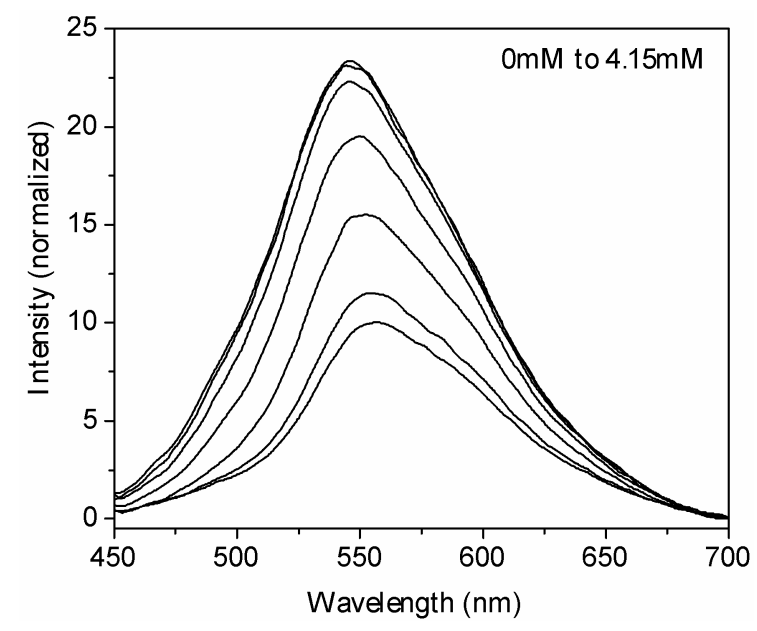

Figure 9. Effect anionic surfactant (SDS) on FL of MEHPPV(60)-g-PAA. The spectra are concentration normalized (1 mM w.r.t. PAA).

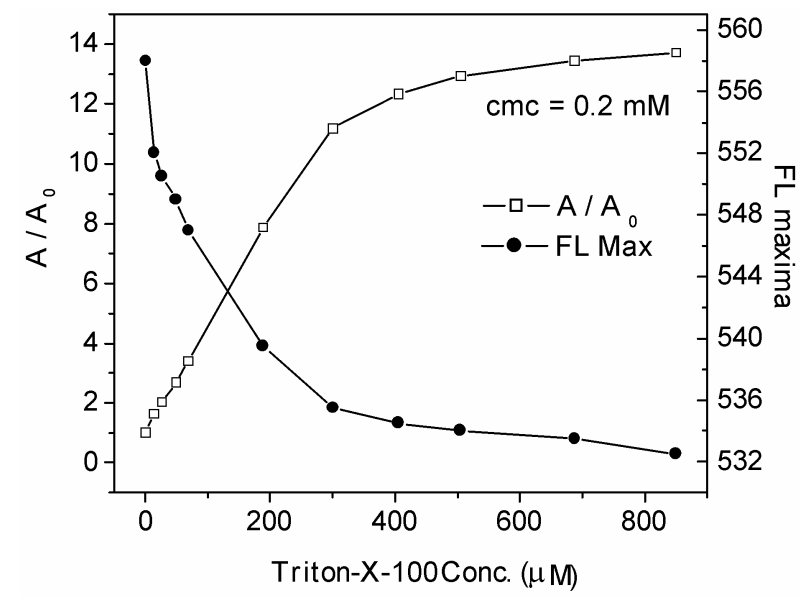

Figure 10. Effect of Triton-X-100 on Fl spectra of MEHPPV(60)-g-PAA.

\subsection{Effect of anionic and nonionic surfactants}

The variation of the FL spectra of MEHPPV-60-gPAA in the presence of an anionic surfactant, SDS (figure 9), surprisingly exhibited a larger variation than of $\mathrm{NaCl} / \mathrm{TEAC}$, even though direct association of the dodecyl sulphonate groups with the anionic PAA tethers is not conceivable. The enhancement is this case, however, is smaller than in the case of the cationic surfactants, even though higher concentrations were used. Although the exact origin of this enhancement remains uncertain, it is possibly related to the interaction of MEHPPV-60-g-PAA with the hydrophobic aggregates formed by SDS.

The effect of a nonionic surfactant, namely Triton-X-100, on the FL spectra of MEHPPV-60-gPAA was even more dramatic, exhibiting more than an order of magnitude increase in the emission intensity. Figure 10 depicts the variations in FL intensity as well as the variation in $\lambda_{\max }$ values as a function of surfactant concentration. This rather unexpected enhancement in FL intensity is similar to the observations made by Burrows et $a l^{29}$ in the case of polyfluorene-based anionic polyelectrolytes. In their case, the dramatic increase was seen only post $\mathrm{CMC}$, and they have ascribed it to the incorporation of their conjugated polyelectrolytes within the micellar core, which in turn they argue leads to a disruption of poorly emissive aggregates. ${ }^{29}$ In our case, however, a significant enhancement occurs at concentrations lower than CMC (CMC of Triton-X-100 is $200 \mu \mathrm{M}$ ); in fact the enhancement is nearly 7 -fold even before CMC is reached. Thus, in our systems the enhancement could originate both due to specific interactions of the surfactants with the polyelectrolyte-conjugated polymer backbone (at lower concentrations, $<\mathrm{CMC}$ ) and due to extension of the MEHPPV-60-g-PAA chains at the micellar interface at higher concentrations ( $>\mathrm{CMC}$ ).

\section{Conclusions}

Grafting polyelectrolytic chains onto the backbone of conjugated polymers, such as MEHPPV, serves as an interesting alternative to the existing approaches that rely on the placement of ionic groups directly on the conjugated polymer backbone. A drawback of the present approach using the dithiocarbamate precursor is the generation of significant amounts of the homopolymer, which proved difficult to remove. The polyelectrolyte grafted conjugated polymers, MEHPPV-60-g-PAA, were soluble in alkaline medium 
and exhibited dramatic increase in the FL intensity in the presence of a variety of surfactants; most significantly in the case of nonionic and cationic surfactants. The increase in intensity is accompanied by a concomitant blue-shift in the emission maximum, and often by the evolution of a shoulder in the blueregion. Due to the presence of segmented conjugation in our grafted MEHPPV derivatives, the variation in the FL spectra can be ascribed to both ineffective energy transfer from shorter chromophores to longer ones within a single chain (due to coil expansion in the presence of the surfactant) and also due to the inhibition of intra-chain inter-chromophore exciton formation; the former explains the evolution of the blue shoulder while the later explains the blue-shift of the $\lambda_{\max }$ and enhanced emission intensity.

\section{Acknowledgements}

We would like to thank the Council for Scientific and Industrial Research (CSIR) and the Ministry of Human Resources Department, New Delhi, for financial support. SR would like to thank the Department of Atomic Energy for an ORT award. KN thanks the CSIR for a fellowship.

\section{References}

1. Burroughes J H, Bradley D D C, Brown A R, Marks R N, Mackay K, Friend R H, Burn P L and Holmes A B 1990 Nature (London) 347539

2. Tessler N $1999 A d v$. Mater. 11363

3. Yu G, Wang J, Mcelvain J and Heeger A J $1998 A d v$. Mater. 101431

4. Brabec C J, Sariciftci N S and Hummelen J C 2001 Adv. Func. Mater. 1115

5. Chen Z, Xue C, Shi W, Luo F-T, Green S, Chen J and Liu H 2004 Anal. Chem. 766513

6. Zhou X-H, Yan J-C and Pei J 2004 Macromolecules 377078

7. Chen L, McBranch D W, Wang H, Helgeson R, Wudl F and Whitten D G 1999 Proc. Natl. Acd. Sci. USA 9612287

8. Liu B and Bazan G C 2004 Chem. Mater. 164467

9. Kim I-B, Wilson J N and Bunz U H F 2005 Chem. Commum. 1273
10. Chen $\mathrm{L}, \mathrm{Xu} \mathrm{S}$, McBranch $\mathrm{D} \mathrm{W}$ and Whitten $\mathrm{D} \mathrm{G}$ $2000 \mathrm{~J}$. Am. Chem. Soc. 1229302

11. Smith R C, Tennyson A G, Lim M H and Lippard S J 2005 Org. Lett. 73573

12. Khan A, Muller S and Hecht S 2005 Chem. Commum. 584

13. Lavigne J J, Broughton D L, Wilson J N, Erdogan B and Bunz U H F 2003 Macromolecules 367409

14. Hong H, Benjamin I, Kirstein S, Avny Y, Neumann $\mathrm{R}$ and Davidov D 1999 Syn. Met. 1021215

15. Pei J, Liu X-L, Yu W-L, Lai Y-H, Niu Y-H and Cao Y 2002 Macromolecules 357274

16. Kim K, Webster S, Levi N, Carroll D L, Pinto M R and Schanze K S 2005 Langmuir 215207

17. Jiang D-L, Choi C-K, Honda K, Li W-S, Yuzawa T and Aida T 2004 J. Am. Chem. Soc. 12612084

18. Kim C K and Kim C 2005 Macromol. Res. 13460

19. Mwaura J K, Pinto M P, Witker D, Ananthakrihsnan N, Schanze K S and Reynolds J R 2005 Langmuir 21 10119

20. Nagesh K and Ramakrishnan S 2005 Synth. Met. 155320

21. Gowri R, Mandal D, Shivkumar B and Ramakrishnan S 1998 Macromolecules 311819

22. Padmanaban $\mathrm{G}$ and Ramakrishnan S $2000 \mathrm{~J}$. Am. Chem. Soc. 1222244

23. Padmanaban G, Nagesh K and Ramakrishnan S 2003 J. Poly. Sci. A41 3929

24. Otsu T and Kuriyama A 1984 Polym. Bull. 11135

25. Otsu T 2000 J. Poly. Sci. A38 2121

26. Perrin D D, Armarego W L F and Perrin D R 1980 Purification of laboratory chemicals 4th edn (Oxford: Pergamon)

27. Nagesh K $2006 \mathrm{PhD}$ thesis Indian Institute of Science, Bangalore

28. Padmanaban G and Ramakrishnan S 2004 J. Phys. Chem. B108 1493

29. Burrows H D, Lobo V M M, Pina J, Ramos M I, Melo J S, Valente A J M, Tapia M J, Pradhan S and Scherf U 2004 Macromolecules 377425

30. Tapia M J, Burrows H D, Valente A J M, Pradhan S, Scherf U, Lobo V M M, Pina J and Melo J S 2005 J. Phys. Chem. B109 19108

31. The CMC's of the all the surfactants were determined using conductometric titration

32. Macknight W J, Ponomarenko E A and Tirrell D A 1998 Acc. Chem. Res. 31781

33. Nörenberg R, Klingler J and Horn D 1999 Angew. Chem. Int. Ed. 381626

34. Deo N, Jockusch S, Turro N J and Somasundaran P 2002 Langmuir 195083

35. Sen S, Dutta P, Sukul D and Bhattacharyya K 2004 J. Phys. Chem. A106 6017 